Trauma Berufskrankh $2005 \cdot 7$ [Suppl 1]:S27-S32 DOI 10.1007/s10039-004-0975-5

(c) Springer Medizin Verlag 2004
Online publiziert: 10. Dezember 2004

P. F. Stahel · M. Infanger - I. M. Bleif · C. E. Heyde - W. Ertel Klinik für Unfall- und Wiederherstellungschirurgie der Charité Universitätsmedizin Berlin, Campus Benjamin Franklin, Berlin

\section{Die palmare winkelstabile Plattenosteosynthese}

\section{Ein neues Konzept zur Versorgung instabiler distaler Radiusfrakturen}

teoporose) und der Ausprägung der dorsalen Trümmerzone - sowie für Komplikationen von Weichteilen und Einschränkungen der Gelenkbeweglichkeit.

\section{Nachteile}

Die negativen Folgen einer konservativen Frakturbehandlung wurden bereits Anfang des 20. Jahrhunderts durch Lorenz Böhler (1885-1973) als „KnochenbruchKrankheit" bezeichnet [6]. Dieses Syndrom ist charakterisiert durch Kontrakturen, Gelenksteife, Muskelatrophie bis hin zur Entwicklung einer Algodystrophie (M. Sudeck), im angelsächsischen Raum auch „complex regional pain syndrome type I“ (CRPS I) genannt [7, 8].

Die Komplikationen der konservativen Therapie haben dazu geführt, dass die Tendenz der Versorgungskonzepte in jüngster Zeit immer mehr in Richtung osteosynthetischer Stabilisierung und frühfunktioneller Nachbehandlung geht $[2,3,9,10,11,12$, 13]. Insbesondere bei Vorliegen definierter Instabilitätkriterien ( $\bullet$ Tabelle 1 ) bei extraartikulären Brüchen sowie bei allen intraartikulären Frakturen mit Gelenkstufenbildung (sog. „Melone-type-Frakturen") stellt sich die Indikation zur operativen Versorgung [14].

\section{Herkömmliche Prinzipien der operativen Versorgung}

Die unterschiedlichen operativen Verfahren zur Stabilisierung distaler Radiusfrakturen umfassen die minimal-invasive KirschnerDraht-Osteosynthese, die externe Fixation sowie die offene Reposition und interne Fixation mit dorsaler oder palmarer Plattenosteosynthese $[2,3,10,12,14,15,16,17,18]$.

Kirschner-Draht-Osteosynthese. Die Kirschner-Draht-Osteosynthese bleibt im Rahmen des Wandels der therapeutischen Strategien in den letzten Jahren [2] eigentlich nur noch der Versorgung pädiatrischer Frakturen und ggf. der auxiliären Stabilisierung einfacher extraartikulärer Frakturen (A2-Typ nach AO) bei Erwachsenen ohne weitere Instabilitätskriterien (- Tabelle 1) vorbehalten.

Externe Fixation. Weiterhin stellt die geschlossene Reposition und Retention im gelenküberbrückenden Fixateur externe den Standard zur temporären Stabilisie-

Tabelle 1

Radiologische Instabilitätskriterien distaler Radiusfrakturen

1 Dorsale Trümmerzone mit Radiusverkürzung $>4 \mathrm{~mm}$

2 Abrissfraktur des Processus styloideus ulnae

3 Dorsale Abkippung des distalen Fragmentes $>20^{\circ}$

4 Abbruch einer palmaren Gelenklippe

5 Palmarkippung des distalen Fragmentes bei schrägem Frakturverlauf eine sekundäre Dislokation - in Abhängigkeit von der Qualität des Knochens (Os- 


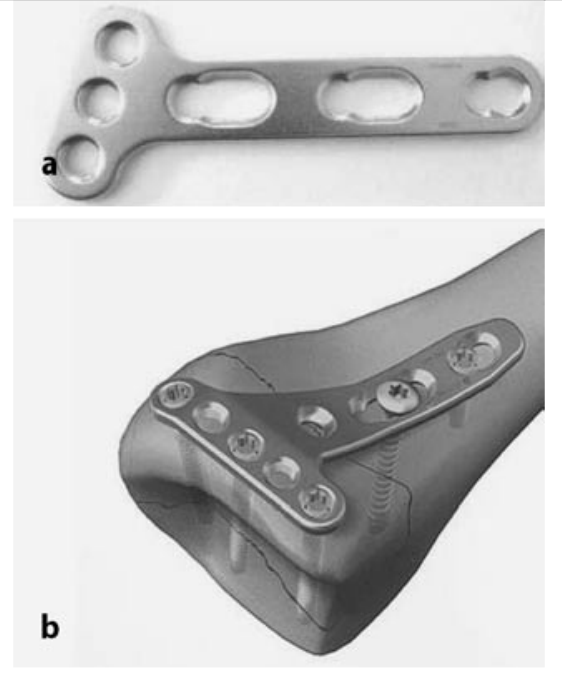

Abb. 1a, b $\Delta$ "Locking compression plate" (LCP) zur winkelstabilen Osteosynthese distaler Radiusfrakturen: 3,5-mm-System (a) und 2,4-mm-/2,7-mm-System (b)

rung von komplexen intraartikulären distalen Radiusfrakturen infolge von Hochrasanzverletzungen oder bei Polytraumapatienten dar $[17,18,19]$. Bei stabiler Fraktur und adäquater Reposition kann die externe Fixation ggf. als definitives Konzept bis zur Ausbehandlung der Fraktur fortgeführt werden $[17,18]$.

Offene Reposition und interne Fixation. Das „klassische“ AO Konzept der offene Reposition und internen Fixation (ORIF) instabiler distaler Radiusfrakturen strebt das Ziel der anatomischen Rekonstruktion der Gelenkflächen, der Restoration der radioulnaren und radiokarpalen Stabilität und der stabilen Retention der Fraktur an [14].

Hierbei wurde seit der Einführung der 3,5-mm-T-Platte für den distalen Radius durch Maurice E. Müller Ende der 196oerJahre das Konzept verfolgt, Frakturen mit dorsaler Dislokation über einen dorsalen Zugang - bzw. bei ventraler Abkippung (sog. „Smith-type“ Frakturen) über einen palmaren Zugang - zu versorgen [14, 20]. Dieses biomechanische Konzept stellte über mehrere Jahrzehnte bis in die heutige Zeit den "golden standard“ der operativen Versorgung distaler Radiusfrakturen dar.

Neben der „klassischen“ T-Platte der AO wurden auch unterschiedliche Formplatten sowie die Doppelplatten-Osteosynthese als erfolgreiches Konzept beschrieben $[2,10,12,20,21]$.
Pi-Platte. Mitte der 199oer-Jahre wurde die erste winkelstabile Platte zur dorsalen Stabilisierung distaler Radiusfrakturen eingeführt (sog. „Pi-plate“ oder „Jupiter-plate"). Während mit dieser Platte erstmals die erheblichen biomechanischen Vorteile der winkelstabilen Osteosynthese durch die erhöhte Ausreißfestigkeit vor allem im osteoporotischen Knochen nachgewiesen werden konnte [22, 23], waren im weiteren Verlauf die klinischen Resultate mit der dorsalen Pi-Platte eher ernüchternd [12].

Das Hauptproblem dieser in Form ei-

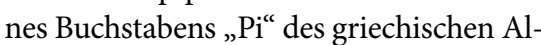
phabets der speziellen Anatomie des distalen Radius angepassten Platte ist die Notwendigkeit eines ausgedehnten dorsalen Zuganges mit Freilegung mehrerer Sehnenfächer sowie die lokale Irritation der Extensorensehnen durch das Osteosynthesematerial [12]. Dies führt bei einem erheblichen Teil der mit einer Pi-Platte versorgten Patienten zu Komplikationen der Sehnenscheiden mit chronischen Schmerzen und Tendovaginitis bis hin zur Strecksehnenruptur durch Abnützung am Implantat $[12,22,24,25,26,27,28,29]$.

\section{Unterfütterung des ossären Defektes.}

Ein weiterer wichtiger Nachteil der konventionellen dorsalen Plattenosteosynthese bei dorsal dislozierten Frakturen, was dem Verletzungsmuster in etwa $90 \%$ aller distalen Radiusfrakturen entspricht [1, 15], ist die Notwendigkeit einer Unterfütterung des ossären Defektes der dorsalen Trümmerzone mit einer Spongiosaplastik oder einem Knochenersatzstoff [12, 30].

Das Standardverfahren durch Unterfüttern des ossären Defekts mit autologer Spongiosa wird allein in Europa jährlich in über 25.00o Fällen durchgeführt, ist aber nach wie vor mit einer bedeutenden Komorbidität im Bereich der Entnahmestelle am Beckenkamm assoziiert, wie Schmerzen, Nachblutung/Hämatome, Infektionen, Verletzung des N. cutaneus femoris lateralis, oder iatrogene Frakturen der Beckenschaufel [31, 32, 33, 34]. Die Gewinnung der autologen Spongiosa - als "golden standard" zur Auffüllung dorsaler Knochendefekte am distalen Radius - bedeutet eine assoziierte Belastung für den Patienten durch den zusätzlichen operativen Zugang, die Verlängerung der Eingriffszeit und das bestehende Zusatzrisiko für Komplikationen im Bereich der Spongiosaentnahmestelle.

Diesbezüglich wurde in der internationalen Literatur über eine Morbidität der Entnahmestelle am Beckenkamm in bis zu 50\% der Fälle berichtet $[31,32,33,34]$. Im Gegensatz dazu werden die nichtautologen Augmentationsformen der biologischen Anforderung einer Knochentransplantation nur teilweise gerecht und gehen ebenfalls mit einer erhöhten Komplikationsrate einher.

Komplikationen. $\mathrm{Zu}$ den bekannten Problemen und Komplikationen einer ,konventionellen" dorsalen Plattenosteosynthese distaler Radiusfrakturen gehören Implantatversagen bei osteoporotischem Knochen, Nichtverheilen der Fraktur durch Kompromittierung der periostalen Durchblutung durch konventionelle Implantate, Strecksehnenbeeinträchtigung durch dorsal eingebrachte Platten, Komplikationen im Bereich der Spongiosaentnahmestelle am Beckenkamm für die dorsale Defektauffüllung am Radius etc.

Mit dem Ziel, diese zu vermeiden, wurde mit der Entwicklung der „locking compression plate" (LCP) durch die $\mathrm{AO}$ im neuen Jahrtausend ein revolutionäres Konzept der Osteosynthese für instabile distale Radiusfrakturen eingeführt $[11,13,35,36$, $37,38,39,40,41]$.

\section{Konzept der winkelstabilen Osteosynthese mit der LCP}

Das Konzept der Winkelstabilität mit den Vorteilen der erhöhten Ausreißfestigkeit der Schrauben und einer verbesserten periostalen Durchblutung durch reduzierten Kontakt der Implantate mit dem Knochen wurde seit den 199oer-Jahren zunehmend in die Entwicklung neuer Implantate integriert [42]. Dies führte über die ersten internen „Platten-Fixateure“ („point contact fixator" oder „PC-Fix“) zur LISS (,less invasive stabilization system") und zur Entwicklung der LCP („locking compression plate“; - Abb. 1; [35, 41, 42]).

Die LCP hat seit der Einführung in prospektive klinische Studien im Jahr 2001 bereits weltweite Verbreitung gefunden $[36,42]$. Bei diesen winkelstabilen Implantaten ist das direkte Anpressen der Platte auf den Knochen zur rigiden Sta- 
Trauma Berufskrankh 2005 - 7[Suppl 1]:S27-S32

DOI 10.1007/s10039-004-0975-5

C) Springer Medizin Verlag 2004
P. F. Stahel · M. Infanger - I. M. Bleif · C. E. Heyde · W. Ertel

\section{Die palmare winkelstabile Plattenosteosynthese. Ein neues Konzept zur Versorgung instabiler distaler Radiusfrakturen}

\section{Zusammenfassung}

Durch die Einführung der „locking compression plate" (LCP) ist die Kombination konventioneller Schrauben mit winkelstabilen Kopfverriegelungsschrauben im selben Implantat möglich geworden. Wir stellen hier ein neues Konzept der winkelstabilen Osteosynthese dorsal abgekippter distaler Radiusfrakturen über einen biologisch unproblematischen palmaren Zugang vor. Die stabile Retention des distalen Fragmentes durch die Kopfverriegelungsschrauben der LCP erlaubt eine frühfunktionelle Nachbehandlung unmittelbar postoperativ ohne Notwendigkeit der auxiliären Gips- retention. Vorteile sind bessere Beweglichkeit im Handgelenk und die reduzierte Inzidenz von Weichteilkomplikationen, wie z. B. der Algodystrophie (CRPS I). Durch die Winkelstabilität der LCP ist die Unterfütterung der dorsalen Trümmerzone mit einer autologen Spongiosaplastik obsolet. Durch die LCP wird eine verminderte Komorbidität durch die Spongiosaentnahme am Beckenkamm, verbesserte periostale Durchblutung durch verminderten Anpressdruck im osteoporotischen Knochen im Vergleich zu herkömmlichen Implantaten erreicht. und signifikant erhöhte Ausreißfestigkeit

\section{Palmar angular-stable plate osteosynthesis: a new concept for treatment of unstable distal radius fractures}

\section{Abstract}

The recent introduction of the locking compression plate (LCP) enables a new and unique combination of angular stable locking head screws with conventional screws in the same implant. The availability of this new plate has lead to a virtual "revolution" in the treatment of unstable distal radius fractures. Here, we present a new concept of palmar plating of dorsally dislocated distal radius fractures with the angular stable $3.5 \mathrm{~mm}$ LCP oblique T-plate. The stable fixation of the distal metaphyseal fragment by locking head screws allows for an early functional rehabilitation without the necessity of auxiliary cast immobilization.
Advantages of this concept include the improved wrist function and range of motion and the decreased risk for soft tissue complications, such as the "complex regional pain syndrome type I" (CRPS I). Furthermore, the angular stability of the LCP eliminates the necessity of additional bone grafting of the dorsal comminution zone, thus preventing additional co-morbidity due to autologous cancellous bone grafting from the iliac crest. In addition, the lack of compression of the periosteum and the increased tear-out resistance of the LCP - particularly in osteoporotic bone - leads to a reduced incidence of adverse events, such as non-union or second-
Dadurch ist das Konzept der palmaren winkelstabilen Plattenosteosynthese zu einem neuen Versorgungs-Standard geworden, der die konventionellen Verfahren wie gelenküberbrückender Fixateur externe und dorsale Plattenosteosynthese aus ihrer führenden Rolle weitgehend verdrängt hat.

\section{Schlüsselwörter}

"Locking compression plate" (LCP) .

Winkelstabile Osteosynthese .

Distale Radiusfrakturen · Palmarer Zugang . Algodystrophie (CRPS I) ary loss of reduction. These significant advantages support the notion of palmar plating with LCP representing an ideal new concept for treatment of unstable distal radius fractures which may replace conventional strategies as a new standard in the near future, such as external fixation or dorsal plating with autologous bone grafting.

\section{Keywords}

Locking compression plate (LCP).

Distal radius fractures - Angular stability .

Palmar approach · Functional rehabilitation 


\section{Neue Osteosynthesetechniken}
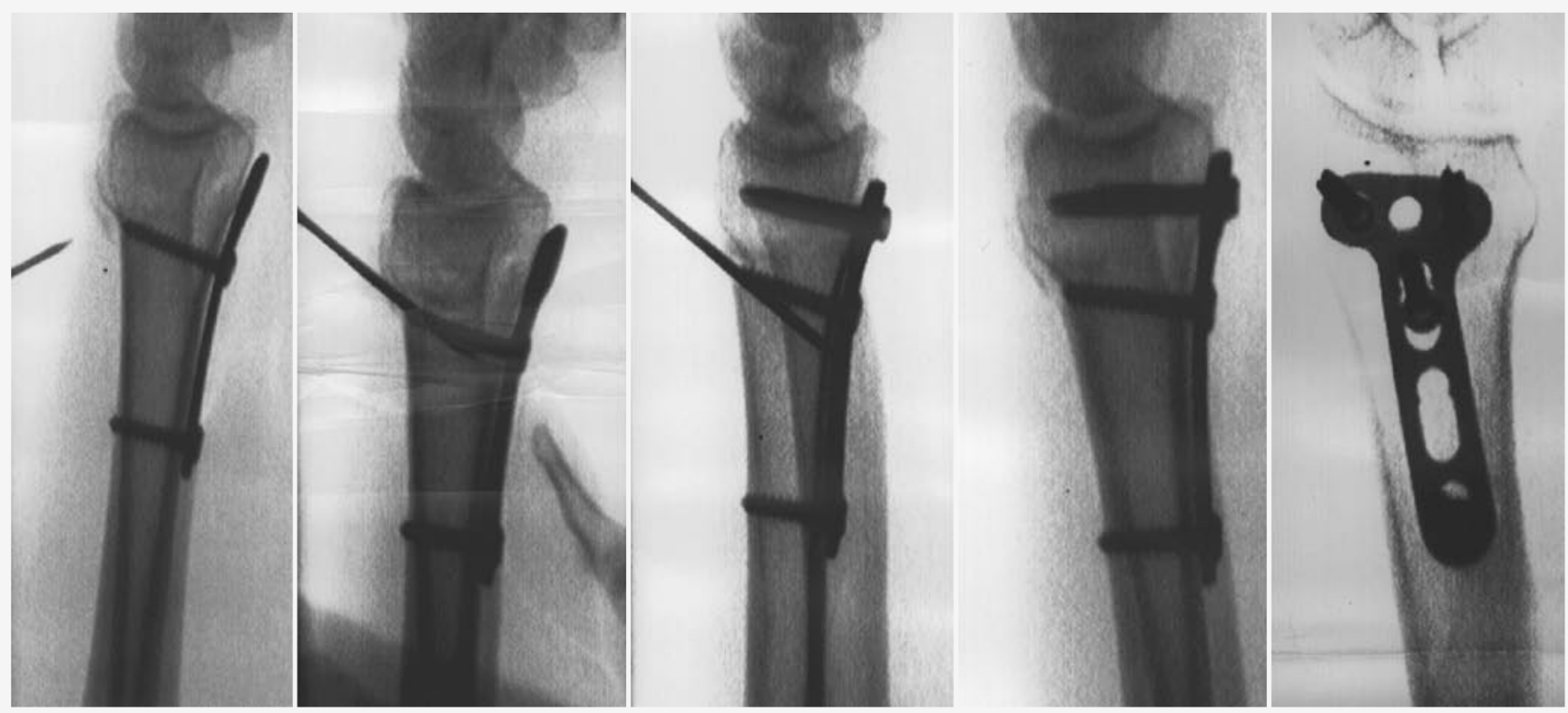

Abb. $2 \Delta$ Repositionsmanöver bei dorsal abgekippter Fraktur: Fixation der 3,5-mm-LCP über palmaren Zugang und Reposition über Ligamentotaxis und modifiziertes perkutanes KapandjiManöver von dorsal
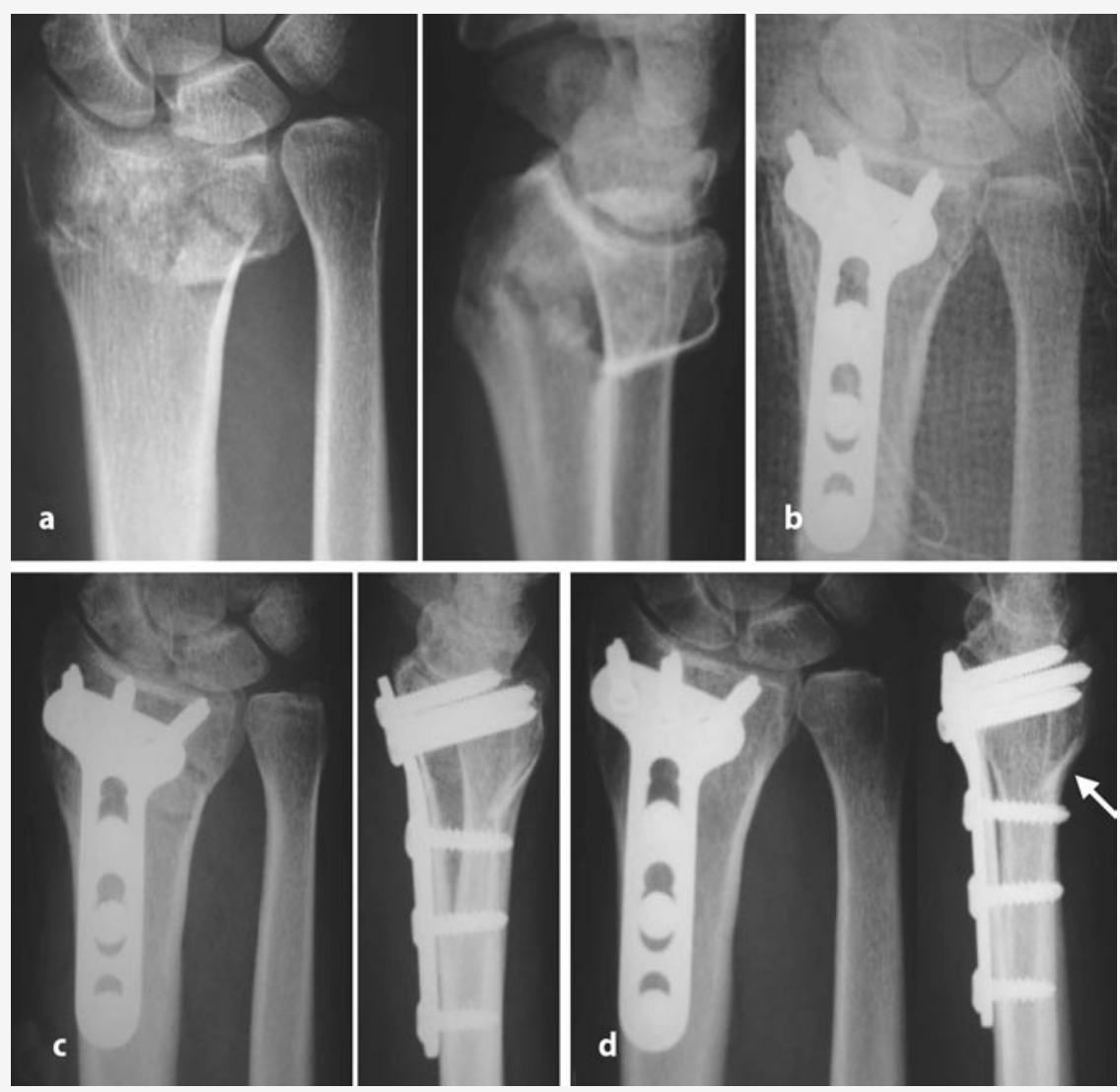

Abb. 3a-d $\Delta$ Fallbeispiel: 45-jährige Patientin mit dorsal disloziertem intraartikulärem Biegungs-I Stauchungsbruch (AO 23-C2) nach Sturz auf die ausgestreckte Hand: Unfallbild (a), postoperative Versorgung (b), Verlaufskontrollen nach 6 Wochen (c) und nach 6 Monaten (d). Kein Nachweis einer sekundären Dislokation der Fraktur trotz frühfunktioneller Nachbehandlung ohne Gipsruhigstellung. Die unversorgte dorsale Trümmerzone ist nach 6 Monaten komplett konsolidiert (Pfeil)
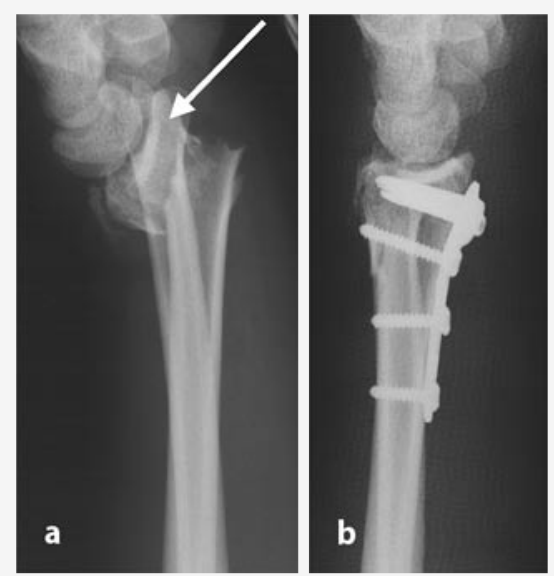

Abb. $4 \triangle$ Distale Radiusfraktur mit dorsale Abkippung und relativ schmalem distalem metaphysärem Fragment (a, Pfeil). Problemlose Retention von palmar winkelstabilen Kopfverriegelungsschrauben (b) 
bilität der Fraktur obsolet [38, 41, 42, 43]. Durch den fehlenden Anpressdruck wird die periostale Blutversorgung weiterhin gewährleistet. Ein zusätzlicher wichtiger Vorteil der Osteosynthese mit winkelstabilen Implantaten stellt die hohe Ausreißfestigkeit im osteoporotischen Knochen dar $[36,38,42]$.

Als Innovation weist die LCP ein kombiniertes Loch aus dem klassischen ovalen Loch zur dynamischen Kompression und dem internen Fixateur-Loch mit winkelstabilem Schraubengewinde auf (• Abb. 1). Das Kombinationsloch erlaubt die freie Wahl des geeigneten Schraubentyps (konventionell vs. winkelstabil) in Abhängigkeit von den individuellen Anforderungen der verschiedenen Frakturen und der unterschiedlichen Knochenqualität. Mehrfache prospektive Mono- und Multicenterstudien sind in den letzten Jahren angelaufen und haben anhand erster Auswertungen die Wertigkeit der LCP zur Versorgung von osteoporotischen Frakturen des distalen Radius und des proximalen Humerus belegt [11, $13,36,44,45,46]$.

\section{Neue Operationstechnik}

Die palmare winkelstabile T-Platten-Osteosynthese ist ein geeignetes Verfahren zur Stabilisierung der instabilen Extensionsfrakturen am distalen Radius, insbesondere der $\mathrm{AO}-23-\mathrm{A}_{3}-/ \mathrm{B}_{3}-/ \mathrm{C}_{1}$ - bis $\mathrm{C}_{3}$ Frakturen. Über den palmaren Zugang, der sich als technisch einfach und komplikationsarm erweist, lassen sich die Frakturen anatomisch reponieren und durch das neue winkelstabile System langfristig stabil fixieren. Das Therapiekonzept basiert auf der hohen Stabilität und Ausreißfestigkeit der winkelstabilen LCP, insbesondere auch bei osteoporotischem Knochen. Die distal in die Platte eingebrachten winkelstabilen Schrauben ermöglichen auch bei dorsal abgekippten distalen Radiusfrakturen mit ausgedehnter Trümmerzone eine hohe Stabilität bei palmar eingebrachter LCP.

Präparation. Der Eingriff erfolgt in Rückenlage mit dem Handtisch und in pneumatischer Oberarmblutleere von 250-280 mmHg. Radiopalmarer Hautschnitt über der Sehne des M. fle- xor carpi radialis (FCR) in einer Länge von ca. 5 cm, mit der Option, den Karpaltunnel spalten zu können. Longitudinale Eröffnung der Unterarmfaszie entsprechend dem palmaren Anteil der Sehnenscheide des FCR, in gleicher Länge und Richtung Durchtrennen des dorsalen Anteils. Stumpfe Präparation radial der Flexoren, welche nach ulnar weggehalten werden. Radialseitige scharfe Ablösung des M. pronator quadratus und Mobilisation desselben nach ulnar. Dabei ergibt sich eine übersichtliche Präparation der Fraktur, vor allem distalseitig, ohne das $\mathrm{Ge}$ lenk zu eröffnen.

Frakturversorgung. Das Konzept der Ligamentotaxis wird zur Frakturreposition unter axialem Zug genutzt. Die Fraktur wird nun mit Kirschner-Drähten der Stärke 1,4 mm durch palmares Eingehen in den Frakturspalt angehoben und vorsichtig reponiert und mit radialseitig perkutan eingebrachten Spickdrähten temporär retiniert. Die winkelstabile LCP wird $15^{\circ}$ palmar vorgebogen, palmar platziert und proximal mit 3,5-mm-Kortikalisschrauben fixiert. Unter leichtem Druck und Gegendruck von dorsal werden nun 2-3 winkelstabile Schrauben in die distalen Fragmente fixiert, welche die Radiusgelenkfläche in der anatomisch korrekten Position halten.

Anschließend werden alle KirschnerDrähte entfernt. In vereinzelten Fällen komplexer intraartikulärer Frakturen vom $\mathrm{C}_{3}$-Typ können auxiliär eingebrachte gelenkrekonstruierende Kirschner-Drähte für 4-6 Wochen in situ belassen werden. Es erfolgt eine frühfunktionelle Nachbehandlung ohne Notwendigkeit der Gipsruhigstellung.

Erfahrungen über $\mathbf{2}$ Jahre. Eigene Erfahrungen mit dieser neuen Technik an über 150 Frakturen mit einer Nachuntersuchungszeit von bis zu 2 Jahren belegen die einfache und komplikationsarme Anwendbarkeit dieser Technik mit langfristiger stabiler Retention der palmaren winkelstabilen Platte ohne Notwendigkeit einer autologen Spongiosaplastik und frühfunktioneller Nachbehandlung mit klinisch exzellenten Resultaten. Die - Abb. 2, 3, 4 zeigen radiologische Fallbeispiele des Versorgungskonzeptes mit der palmaren 3,5mm-LCP.

\section{Fazit für die Praxis}

Die Vorteile der winkelstabilen Plattenosteosynthese distaler Radiusfrakturen mit der LCP wie der Erhalt der periostalen Durchblutung, die erhöhte Ausreißfestigkeit und die variable Möglichkeit des Einsatzes konventioneller und winkelstabiler Schrauben erlauben eine breiten Einsatz dieser Platte auch über das Spektrum der herkömmlichen Indikationen hinaus.

Die ersten Ergebnisse legen den Schluss nahe, dass auch bei Defektzonen nach reponierten eingestauchten Brüchen Spongiosatransplantationen in der überwiegenden Zahl der Fälle durch die hohe Stabilität des Konstruktes entbehrlich sind. Dadurch entfällt mit diesem neuen Versorgungskonzept auch die Morbidität der Spongiosaentnahme am Beckenkamm. Die hohe Stabilität des winkelstabilen Platten-/Schraubenkopfverbundes erlaubt die Versorgung der viel häufigeren Extensionsfrakturen des distalen Radius, vor allem auch bei osteoporotischem Knochen, vom palmaren Zugang aus.

Durch diesen anatomisch unproblematischen Zugang können die bekannten Langzeitprobleme vermieden werden, wie Komplikationen im Bereich der Strecksehnengleitlager. Die frühfunktionelle Nachbehandlung ohne Notwendigkeit der Gipsruhigstellung verhindert die Entwicklung der herkömmlichen „Frakturkrankheit" und des CRPS I.

\section{Korrespondierender Autor}

PD Dr. P. F. Stahel

Klinik für Unfall- und

Wiederherstellungschirurgie der Charité Universitätsmedizin Berlin,

Campus Benjamin Franklin,

Hindenburgdamm 30, 12200 Berlin

E-Mail: pfstahel@aol.com

\section{Abbildungsnachweis}

Wir danken der Fa. Synthes, Bochum, für die zur Verfügung gestellten Abbildungen der LCP in - Abb. 1.

Interessenkonflikt: Der korrespondierende Autor versichert, dass keine Verbindungen mit einer Firma, deren Produkt in dem Artikel genannt ist, oder einer Firma, die ein Konkurrenzprodukt vertreibt, bestehen. 


\section{Literatur}

1. Rikli D, Regazzoni P (1999) Distale Radiusfrakturen. Schweiz Med Wochenschr 129: 776-85

2. Wiemer P, Köster G, Felderhoff J, Weber U (1999) Frakturen am distalen Radius: Wandel der therapeutischen Strategien. Orthopäde 28: 846-852

3. Ruch DS, Weiland AJ, Wolfe SW, Geissler WB, Cohen MS, Jupiter JB (2004) Current concepts in the treatment of distal radial fractures. Instr Course Lect 53: 389-401

4. Oestern HJ (1999) Distale Radiusfrakturen - Teil I: Grundlagen und konservative Therapie. Chirurg 70: 1180-1192

5. Smilovic J, Bilic R (2003) Conservative treatment of extra-articular Colles' type fractures of the distal radius: prospective study. Croat Med J 44: 740-745

6. Szyskowitz R (2002) Lorenz Böhler. AO Dialogue 15: 16-17

7. Schürmann M, Gradl G, Zaspel I, Kayser M, Lohr P, Andress HJ (2000) Peripheral sympathetic function as a predictor of complex regional pain syndrome type I (CRPS I) in patients with radial fracture. Auton Neurosci 86: 127-134

8. Gradl G, Steinborn M, Wizgall I, Mittlmeier T, Schürmann M (2003) Das akute CRPS I (Morbus Sudeck) nach distaler Radiusfraktur: Methoden der Frühdiagnostik. Zentralbl Chir 128: 1020-1026

9. Rikli DA, Regazzoni P (1996) Fractures of the distal end of the radius treated by internal fixation and early function. A preliminary report of 20 cases. $J$ Bone Joint Surg Br 78: 588-592

10. Jakob M, Rikli DA, Regazzoni P (2000) Fractures of the distal radius treated by internal fixation and early function. A prospective study of 73 consecutive patients. J Bone Joint Surg Br 82: 340-344

11. Rikli DA, Babst R (2003) Neue Prinzipien der operativen Behandlung von distalen Radiusfrakturen: winkelstabile Implantate. Ther Umsch 60: 745-50

12. Hahnloser D, Platz A, Amgwerd M, Trentz O (1999) Internal fixation of distal radius fractures with dorsal dislocation: pi-plate or two 1/4 tube plates? A prospective randomized study. J Trauma 47: 760-765

13. Schütz M, Kolbeck S, Spranger A, Arndt-Kolbeck M, Haas NP (2003) Die winkelstabile palmare Plattenosteosynthese bei der dorsal dislozierten distalen Radiusfraktur: Anwendung und erste klinische Erfahrungen. Zentralbl Chir 128: 997-1002

14. Oestern HJ (1999) Distale Radiusfrakturen - Teil II: Operative Therapien. Chirurg 70: 1381-1394

15. Lindenmann-Sperfeld L, Pilz F, Marintschev I, Otto W (2003) Der distale Speichenbruch: minimalinvasive Kirschnerdrahtosteosynthese - Indikation und Ergebnisse. Chirurg 74: 1000-1008

16. Rikli DA, Kupfer K, Bodoky A (1998) Long-term results of the external fixation of distal radius fractures. J Trauma 44: 970-976

17. Rikli DA, Rosenkranz J, Regazzoni P (2003) Complex fractures of the distal radius. Eur J Trauma 29: 199-207

18. Rikli DA, Regazzoni P, Babst R (2003) Management komplexer distaler Radiusfrakturen. Zentralbl Chir 128: 1008-1013

19. Jupiter JB (1997) Complex articular fractures of the distal radius: classification and management. J Am Acad Orthop Surg 5: 119-129

20. Felderhoff J, Wiemer P, Dronsella J, Weber U (1999) Operative Versorgung der distalen, instabilen Radiusfraktur mit der dorsalen/palmaren Abstützplatte. Orthopäde 28: 853-863

21. Rikli DA, Regazzoni P, Babst $R$ (2003) Die dorsale Doppelplattenosteosynthese am distalen Radius: ein biomechanisches Konzept und dessen klinische Realisation. Zentralbl Chir 128: 1003-1007
22. Ring D, Jupiter JB, Brennwald J, Büchler U, Hastings H 2nd (1997) Prospective multicenter trial of a plate for dorsal fixation of distal radius fractures. Hand Surg [Am] 22: 777-784

23. Campbell DA (2000) Open reduction and internal fixation of intra-articular and unstable fractures of the distal radius using the $\mathrm{AO}$ distal radius plate. $J$ Hand Surg (Brit) 25: 528-534

24. Kambouroglou GK, Axelrod TS (1998) Complications of the AO/ASIF titanium distal radius plate system (pi plate) in internal fixation of the distal radius. J Hand Surg (Am) 23: 737-741

25. Rozental TD, Beredjiklian PK, Bozentka DJ (2003) Functional outcome and complications following two types of dorsal plating for unstable fractures of the distal part of the radius. J Bone Joint Surg Am 85-A: 1956-1960

26. Herron M, Faraj A, Craigen MA (2003) Dorsal plating for displaced intra-articular fractures of the distal radius. Injury 34: 497-502

27. Chiang PP, Roach S, Baratz ME (2002) Failure of a retinacular flap to prevent dorsal wrist pain after titanium Pi plate fixation of distal radius fractures. J Hand Surg [Am] 27: 724-728

28. Schnur DP, Chang B (2000) Extensor tendon rupture after internal fixation of a distal radius fracture using a dorsally placed $\mathrm{AO} / \mathrm{ASIF}$ titanium pi plate. Ann Plast Surg 44: 564-566

29. Nunley JA, Rowan PR (1999) Delayed rupture of the flexor pollicis longus tendon after inappropriate placement of the pi plate on the volar surface of the distal radius. J Hand Surg (Am) 24: 1279-1280

30. Cassidy C, Jupiter JB, Cohen M et al. (2003) Norian SRS cement compared with conventional fixation in distal radial fractures. A randomized study. $J$ Bone Joint Surg Am 85-A: 2127-2137

31. Arrington ED, Smith WJ, Chambers HG, Bucknell AL, Davino NA (1996) Complications of iliac crest bone graft harvesting. Clin Orthop 329: 300-309

32. Banwart JC, Asher MA, Hassanein RS (1995) Iliac crest bone graft harvest donor site morbidity: a statistical evaluation. Spine 20: 1055-1060

33. Dutting A, Thomas W, Lorenz H, Holst A (1988) Komplikationen nach autologer Knochentransplantation am Entnahmeort. Z Orthop Ihre Grenzgeb 126: 44-47

34. Fowler BL, Dall BE, Rowe DE (1995) Complications associated with harvesting autogenous bone graft. Am J Orthop 24: 895-903

35. Sommer C (2003) Locking compression plate. Injury 34 [Suppl] 2: B4-5

36. Sommer C, Gautier E, Muller M, Helfet DL, Wagner M (2003) First clinical results of the Locking Compression Plate (LCP). Injury 34 [Suppl] 2: B43-54

37. Leung F, Zhu L, Ho H, Lu WW, Chow SP (2003) Palmar plate fixation of $\mathrm{AO}$ type $\mathrm{C} 2$ fracture of distal radius using a locking compression plate: a biomechanical study in a cadaveric model. J Hand Surg [Br] 28: 263-266

38. Stahel PF, Ertel W (2002) Konzept der winkelstabilen Plattenosteosynthese mit der LCP. In: Messmer W (Hrsg) Was gibt es Neues in der Chirurgie? Bd XII. Ecomed, Landsberg, S 1-3

39. Schupp A, Tuttlies C, Mohlig T, Siebert HR (2003) Der distale Speichenbruch: winkelstabile Osteosynthese mit $2.4 \mathrm{~mm}$ Formplatten - ist der Aufwand gerechtfertigt? Chirurg 74: 1009-1017

40. Thielke KH, Wagner T, Bartsch S, Echtermeyer V (2003) Winkelstabile, volare Plattenosteosynthese komplexer artikulärer Frakturen am distalen Radius: Lösung einer Problemfraktur? Chirurg 74: 1057-1063

41. Schütz M, Sudkamp NP ( 2003) Revolution in plate osteosynthesis: new internal fixator systems. J Orthop Sci 8: 252-258
42. Wagner M (2003) General principles for the clinical use of the LCP. Injury 34 [Suppl] 2: B31-42

43. Stoffel K, Dieter U, Stachowiak G, Gachter A, Kuster MS (2003) Biomechanical testing of the LCP - how can stability in locked internal fixators be controlled? Injury 34 [Suppl] 2: B11-19

44. Krimmer $\mathrm{H}$, Pessenlehner $\mathrm{C}$, Hasselbacher $\mathrm{K}$, Meier M, Roth F, Meier R (2004) Palmare winkelstabile Plattenosteosynthese der instabilen distalen Radiusfraktur. Unfallchirurg 107: 460-467

45. Walz M, Kolbow B, Auerbach F (2004) Was leistet die winkelstabile Plattenosteosynthese bei der distalen Radiusfrakture des alten Menschen? Unfallchirurg 107: 664-670

46. Lill H, Hepp P, Rose T, König K, Josten C (2004) Die winkelstabile Plattenosteosynthese (LPHP) proximaler Humerusfrakturen über den kleinen anterolateralen Delta-Splitting-Zugang: Technik und erste Ergebnisse. Zentralbl Chir 129: 43-48 